\title{
Adoption of routine telemedicine in Norwegian hospitals: progress over 5 years

Paolo Zanaboni ${ }^{1 *}$ and Richard Wootton ${ }^{1,2}$

\begin{abstract}
Background: Although Norway is well known for its early use of telemedicine to provide services for people in rural and remote areas in the Arctic, little is known about the pace of telemedicine adoption in Norway. The aim of the present study was to explore the statewide implementation of telemedicine in Norwegian hospitals over time, and analyse its adoption and level of use.

Methods: Data on outpatient visits and telemedicine consultations delivered by Norwegian hospitals from 2009 to 2013 were collected from the national health registry. Data were stratified by health region, hospital, year, and clinical specialty.

Results: All four health regions used telemedicine, i.e. there was $100 \%$ adoption at the regional level. The use of routine telemedicine differed between health regions, and telemedicine appeared to be used mostly in the regions of lower centrality and population density, such as Northern Norway. Only Central Norway seemed to be atypical. Twenty-one out of 28 hospitals reported using telemedicine, i.e. there was $75 \%$ adoption at the hospital level. Neurosurgery and rehabilitation were the clinical specialties where telemedicine was used most frequently. Despite the growing trend and the high adoption, the relative use of telemedicine compared to that of outpatient visits was low.

Conclusions: Adoption of telemedicine is Norway was high, with all the health regions and most of the hospitals reporting using telemedicine. The use of telemedicine appeared to increase over the 5-year study period. However, the proportion of telemedicine consultations relative to the number of outpatient visits was low. The use of telemedicine in Norway was low in comparison with that reported in large-scale telemedicine networks in other countries. To facilitate future comparisons, data on adoption and utilisation over time should be reported routinely by statewide or network-based telemedicine services.
\end{abstract}

Keywords: Telemedicine, Telehealth, Adoption, Implementation, Barriers

\section{Background}

Telemedicine can improve access to healthcare services, especially in sparsely populated and less developed regions, by facilitating contact between patients and providers. Telemedicine has been widely tested over the past 20 years and represents a viable and significant adjunct to the delivery of healthcare [1]. However, adoption into routine practice has been slower than anticipated [2], and evidence for its effectiveness [3, 4] and cost-effectiveness [5] is still limited. Nevertheless, results are improving and several telemedicine applications appear to be promising

\footnotetext{
* Correspondence: Paolo.Zanaboni@ehealthresearch.no

${ }^{1}$ Norwegian Centre for E-health Research, University Hospital of North

Norway, P.O. Box 359038 Tromsø, Norway

Full list of author information is available at the end of the article
}

candidates for widespread use [6]. The widespread deployment of telemedicine might improve quality of life, raise productivity in the health sector [7], avoid travel to underserved populations [8], and contribute to the sustainability of national health systems [9].

Norway has 5 million inhabitants who are spread over nearly 400,000 square kilometres, making it one of the most sparsely populated countries in Europe [10]. The responsibility for specialist care lies with the state, administered by four Regional Health Authorities (Northern, Central, Western, and South-Eastern Norway). Each region operates a number of public hospitals (Fig. 1). Municipalities are responsible for primary care. Private specialist health facilities are invited as partners to the 


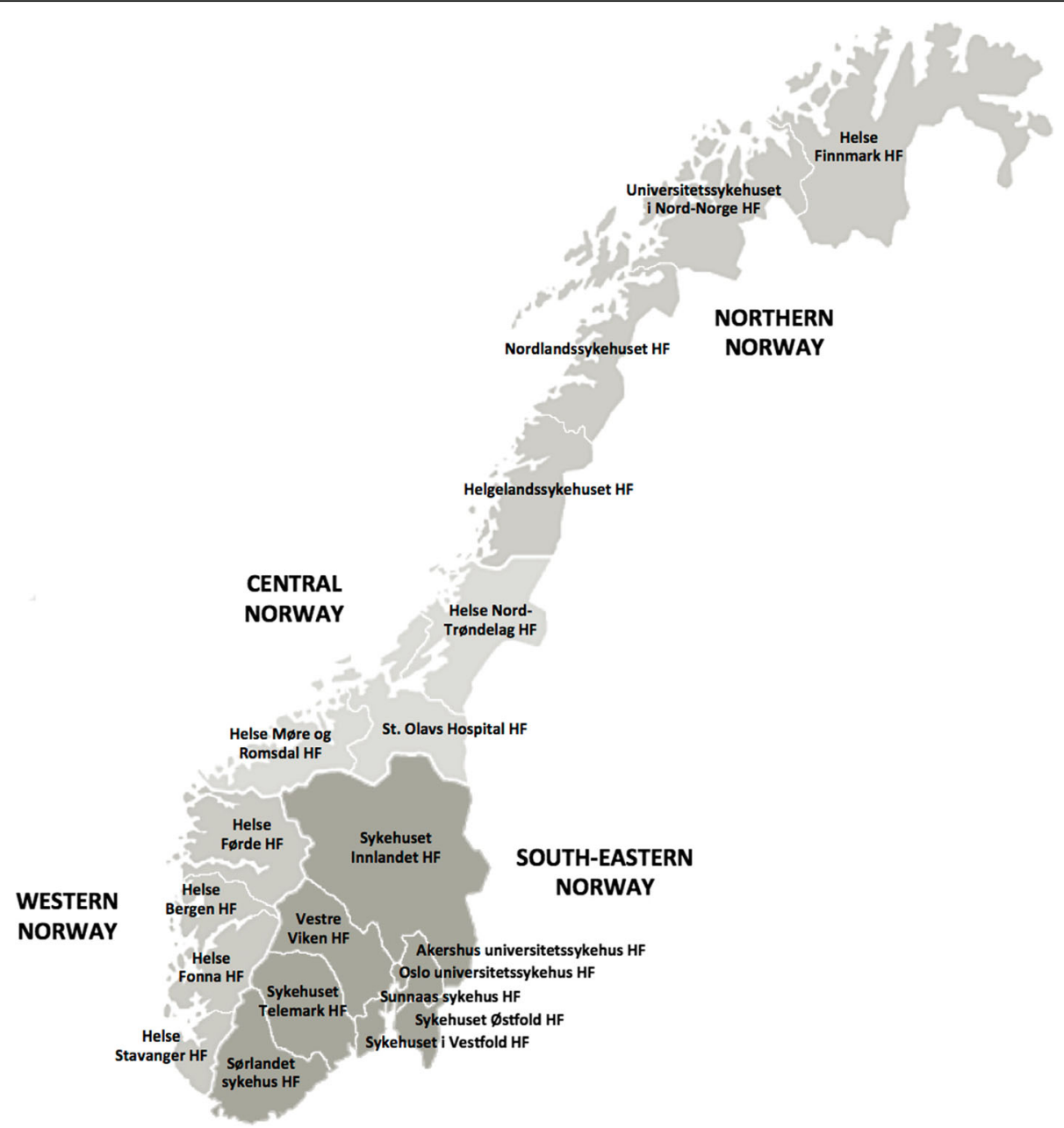

Fig. 1 Health regions and public hospitals in Norway

system on a contractual basis [11]. Despite having one of the highest densities of physicians in Europe, Norway still struggles to ensure geographical and social equity in access to healthcare [12].

Norway is well known for its early adoption of telemedicine to provide services for the population in rural and remote areas in the Arctic [13]. Telemedicine applications have been tested since the early 1990s in the form of pilot projects or small-scale services, some of which have become fully operational [14]. Telemedicine was initially provided as a routine service only to a minor degree, with variations between health regions, but gradually several telemedicine services became ready for largescale implementation [15]. Recently, adoption of telemedicine was reported in all health regions and most hospitals in Norway. However, the level of use was low [16].

Providing access to telemedicine does not mean that the services will be used to capacity [17]. More efforts are required internationally to provide evidence and data about the deployment of telemedicine [7]. The aim of the present study was to explore the statewide implementation of telemedicine services in Norway over time, to analyse the adoption and level of use of telemedicine by health region, by hospital, and by clinical specialty, and to examine the hypothesis that routine telemedicine is mainly used to increase access to healthcare services in remote areas. A secondary aim was to perform an international comparison of the level of telemedicine activity in Norway with other statewide telemedicine networks.

\section{Methods \\ Data collection}

Data on the use of routine telemedicine in Norwegian hospitals were collected from the Norwegian Patient Registry (NPR). The NPR is the central health registry created in 1997 by the Norwegian Directorate of Health to provide data for planning, evaluation, and financing of publicly funded specialised healthcare, as well as for medical and health services research [11]. Data registered 
in the NPR cover inpatient and outpatient care delivered by publicly funded hospitals. Only telemedicine consultations for which hospitals are reimbursed are included. In Norway, a 'telemedicine consultation' is defined as the use of videoconferencing to perform an outpatient consultation, examination, or treatment at a distance. To be registered as a telemedicine activity, a consultation must occur: a) via videoconferencing equipment, meaning that patient and health personnel can see each other through video transmission, $b$ ) in real-time, c) between the patient and at least one health professional, of whom at least one is a doctor, from two different physical locations [18]. The use of store-and-forward telemedicine, including the transmission of still images or remote monitoring of a patient's health parameters, is not covered by any reimbursement scheme in Norway. Contacts occurring by telephone, SMS, or similar means are not considered to be telemedicine consultations. The reimbursement for a telemedicine consultation is equal to that of a traditional outpatient visit.

We sent a formal request to the NPR in April 2014 to obtain data on the telemedicine consultations delivered by Norwegian hospitals from 2009 to 2013. Data related to the outpatient visits were also collected as a means of comparing telemedicine activity with overall hospital activity. The study did not involve human participants, and no personally identifiable data related to individuals were collected. Ethics approvals from the Regional Ethics Committees and informed consents were therefore not required, according to the Norwegian Health Research Act and the Personal Data Act. The Norwegian Directorate of Health approved the request and delivered completed data in November 2014.

\section{Data analysis}

Outpatient visits and telemedicine consultations were stratified by health region, hospital, year, and clinical specialty. Adoption was expressed as the percentage of the number of adopters over the potential users [19]. Since telemedicine can be used to replace outpatient visits, the proportion of telemedicine consultations over the number of outpatient visits was also calculated. The remoteness of each health region was measured through two indexes used to assess the peripherality of Norwegian municipalities: the centrality index (scored 0-20) and the population index (scored 0-10) [20]. Centrality describes the geographic location of a municipality based on the size of the largest urban centre that can be reached within a given travel time. The population index is based on the population density of a municipality. Low values correspond to more isolated and less populated areas, respectively. The indexes for each health region were calculated as the median of the values of all municipalities belonging to that region. Hospitals were arbitrarily stratified by size according to the number of outpatient visits delivered in 2013. Small hospitals had less than 50,000 outpatient visits per year, medium hospitals had 50,000 to 200,000 outpatient visits, while large hospitals had more than 200,000 outpatient visits. Clinical specialties were also arbitrarily stratified by size according to the number of outpatient visits delivered in 2013. Specialties with less than 50,000 outpatient visits per year were considered as low activity, specialties with medium activity had between 50,000 and 200,000 yearly outpatient visits, while high activity was considered as more than 200,000 outpatient visits.

There is a lack of agreed standard measures to calculate telemedicine activity, which makes international comparisons problematic [17]. The number of consultations per site per week has been proposed as a metric to measure telemedicine service use [21]. However, sites may differ in terms of healthcare providers and population served. We selected studies reporting telemedicine activity as the number of telemedicine consultations per year, and compared that to the population served by each telemedicine network. We then calculated the pro capita rate of telemedicine usage.

\section{Results \\ Adoption and use per health region}

Table 1 summarises the number of outpatient visits and telemedicine consultations in publicly funded Norwegian hospitals from 2009 to 2013. The number of outpatient visits increased steadily over the 5-year period and in 2013 there were $11.8 \%$ more outpatient visits than in 2009 . Growth differed from region to region. The highest growth was recorded in Western Norway and Central Norway, with rates of $16.8 \%$ and $15.8 \%$, respectively. Growth rates were lower in South-Eastern Norway (9.5\%) and Northern Norway (9.4\%).

All four health regions reported the use of telemedicine during the 5 -year period, i.e. there was $100 \%$ adoption at the regional level. However, there was a decline in the overall number of telemedicine consultations from 2009 to 2010 (Fig. 2). After 2010 there was a steady increase until 2013. Overall, the number of telemedicine consultations in 2013 was $4.9 \%$ higher than in 2009.

The use of routine telemedicine differed between the health regions (Fig. 2). Western Norway was the only region in which the number of telemedicine consultations increased continually during the study period. In 2013 there were six times more consultations compared to 2009. In the other three regions there was a decline from 2009 to 2010, and then a stable use of routine telemedicine until 2013. Northern Norway, the region delivering most of the telemedicine consultations in 2009, had only half of the consultations in 2010. Western Norway only contributed to the consistent growth characterising 
Table 1 Outpatient visits and telemedicine consultations in the period 2009-2013 in the four health regions in Norway

\begin{tabular}{|c|c|c|c|c|c|c|c|c|c|c|c|c|}
\hline Health region & $\begin{array}{l}\text { Centrality } \\
(0-20)^{\mathrm{a}}\end{array}$ & $\begin{array}{l}\text { Population } \\
(0-10)^{\mathrm{a}}\end{array}$ & $\begin{array}{l}\text { Outpatient } \\
\text { visits (2009) }\end{array}$ & $\begin{array}{l}\text { Outpatient } \\
\text { visits (2010) }\end{array}$ & $\begin{array}{l}\text { Outpatient } \\
\text { visits (2011) }\end{array}$ & $\begin{array}{l}\text { Outpatient } \\
\text { visits (2012) }\end{array}$ & $\begin{array}{l}\text { Outpatient } \\
\text { visits (2013) }\end{array}$ & $\begin{array}{l}\text { Telemedicine } \\
\text { consultations } \\
\text { (2009) }\end{array}$ & $\begin{array}{l}\text { Telemedicine } \\
\text { consultations } \\
\text { (2010) }\end{array}$ & $\begin{array}{l}\text { Telemedicine } \\
\text { consultations } \\
\text { (2011) }\end{array}$ & $\begin{array}{l}\text { Telemedicine } \\
\text { consultations } \\
\text { (2012) }\end{array}$ & $\begin{array}{l}\text { Telemedicine } \\
\text { consultations } \\
\text { (2013) }\end{array}$ \\
\hline Western Norway & 10 & 0.80 & 879,911 & 930,840 & 947,303 & 994,769 & $1,027,463$ & $240(0.03 \%)$ & $246(0.03 \%)$ & $821(0.09 \%)$ & $1586(0.16 \%)$ & $1686(0.16 \%)$ \\
\hline Central Norway & 11 & 0.50 & 695,162 & 724,617 & 763,467 & 784,757 & 804,753 & $448(0.06 \%)$ & $23(0.00 \%)$ & $1(0.00 \%)$ & $0(0.00 \%)$ & $32(0.00 \%)$ \\
\hline Northern Norway & 4 & 0.20 & 470,078 & 484,151 & 502,839 & 515,029 & 514,316 & 1739 (0.37 \%) & $876(0.18 \%)$ & $986(0.20 \%)$ & $955(0.19 \%)$ & 991 (0.19\%) \\
\hline South-Eastern Norway & 14 & 1.30 & $2,573,532$ & $2,625,076$ & $2,711,593$ & $2,783,087$ & $2,819,054$ & $318(0.01 \%)$ & $41(0.00 \%)$ & $19(0.00 \%)$ & 159 (0.01 \%) & $170(0.01 \%)$ \\
\hline Total & 12 & 0.6 & $4,618,683$ & $4,764,684$ & $4,925,202$ & $5,077,642$ & $5,165,586$ & 2745 (0.06 \%) & $1186(0.02 \%)$ & $1827(0.04 \%)$ & $2700(0.05 \%)$ & $2879(0.06 \%)$ \\
\hline
\end{tabular}

Values are expressed as median

Values in brackets (\%) represent the percentage of telemedicine consultations compared to the number of outpatient visits, by year 


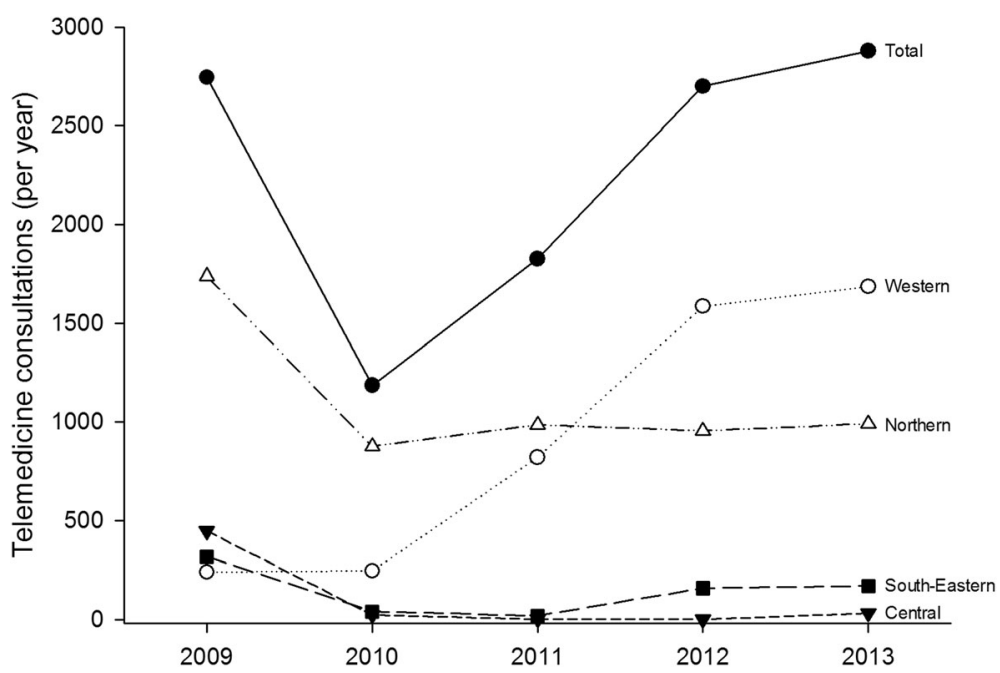

Fig. 2 Telemedicine consultations in the period 2009-2013 in Norway and in the four health regions

the period from 2010 to 2013, thus becoming the region delivering most of the telemedicine consultations.

The use of routine telemedicine seemed to be higher in regions characterised by a lower centrality (Table 1). Similarly, telemedicine appeared to be used to a greater degree in scarcely populated regions. Central Norway seemed to be atypical, using telemedicine less than expected in relation to centrality and population.

\section{Adoption and use per publicly funded hospital}

The number of outpatient visits grew for almost all hospitals over the 5-year period (Table 2). Growth rates ranged from $3.1 \%$ to $63.1 \%$. Only two hospitals had a change lower than $1 \%$.

Twenty-one out of 28 hospitals reported that they had used telemedicine in at least one year during the period 2009-2013, i.e. there was a $75 \%$ adoption at the hospital level. However, not all hospitals used telemedicine continuously over the study period. The number of hospitals reporting telemedicine consultations was 14 in 2010, 15 in 2009 and 2011, and 16 in 2012 and 2013. Telemedicine usage (Fig. 3) and growth (Fig. 4) did not appear to be related to hospital size.

Eleven hospitals delivered more than 50 consultations for at least one year from 2009 to 2013. All the four hospitals in Northern Norway were active in delivering telemedicine over the 5-year study. Three hospitals increased steadily their activity over the period, 7 experienced a decline, while 1 had a variable trend. Helse Stavanger had a large increase of telemedicine activity, with a level in 2013 more than 12 times higher than in 2009 , compared to a growth rate of $22.5 \%$ for outpatient visits. This hospital is mainly responsible for the growth trend characterizing Western Norway and the overall national trend as well. Helse Finnmark also increased considerably its telemedicine activity, with a level in 2013 more than 4 times higher than in 2009. This is a medium-sized hospital whose overall outpatient visits grew only by $7.3 \%$ during the same period. Finally, Sunnaas sykehus is a small-sized hospital specialised in rehabilitation. This hospital did not have any telemedicine consultations in 2009, very few in 2010 and 2011, while in 2012 and 2013 the activity was much higher.

Comparing the number of telemedicine consultations to the number of outpatient visits, Sunnaas sykehus was the hospital which performed best, reporting in 2013 a relative use of telemedicine of $3.51 \%$ of all outpatient activity, consisting mainly of rehabilitation visits. That is, the hospital has been replacing outpatient face-to-face visits with remote consultations performed via videoconferencing. Helse Stavanger, the most active hospital delivering telemedicine, reached a relative use of $0.58 \%$ of the overall outpatient activity. Despite this remarkable growth, the level was still low compared to the number of outpatient visits, indicating great potential for using telemedicine to replace traditional outpatient visits. Of the other hospitals, Universitetssykehuset i Nord-Norge had a modest relative use of telemedicine in 2009 (0.61 \%) compared to outpatient visits. However, this declined in the following years.

\section{Adoption and use per clinical specialty}

The results show that the use of routine telemedicine differed significantly from region to region, and was only used by some of the Norwegian hospitals. Table 3 shows the overall activity in terms of outpatient visits and telemedicine consultations in the period from 2009 to 2013 stratified by clinical specialty. Data are ordered by relative use of telemedicine compared to the overall outpatient activity in the final year, that is 
Table 2 Outpatient visits and telemedicine consultations in the period 2009-2013 in the publicly funded hospitals in Norway

\begin{tabular}{|c|c|c|c|c|c|c|c|c|c|c|c|}
\hline Hospital & Size & $\begin{array}{l}\text { Outpatient } \\
\text { visits (2009) }\end{array}$ & $\begin{array}{l}\text { Outpatient } \\
\text { visits (2010) }\end{array}$ & $\begin{array}{l}\text { Outpatient } \\
\text { visits (2011) }\end{array}$ & $\begin{array}{l}\text { Outpatient } \\
\text { visits (2012) }\end{array}$ & $\begin{array}{l}\text { Outpatient } \\
\text { visits (2013) }\end{array}$ & $\begin{array}{l}\text { Telemedicine } \\
\text { consultations } \\
\text { (2009) }\end{array}$ & $\begin{array}{l}\text { Telemedicine } \\
\text { consultations } \\
(2010)\end{array}$ & $\begin{array}{l}\text { Telemedicine } \\
\text { consultations } \\
(2011)\end{array}$ & $\begin{array}{l}\text { Telemedicine } \\
\text { consultations } \\
\text { (2012) }\end{array}$ & $\begin{array}{l}\text { Telemedicine } \\
\text { consultations } \\
\text { (2013) }\end{array}$ \\
\hline Western Norway & & 879,911 & 930,840 & 947,303 & 994,769 & $1,027,463$ & $240(0.03 \%)$ & $246(0.03 \%)$ & $821(0.09 \%)$ & $1586(0.16 \%)$ & $1686(0.16 \%)$ \\
\hline Helse Stavanger HF & Large & 236,601 & 274,315 & 268,052 & 279,797 & 289,860 & $124(0.05 \%)$ & $201(0.07 \%)$ & $806(0.30 \%)$ & 1583 (0.57 \%) & $1684(0.58 \%)$ \\
\hline Helse Fonna HF & Medium & 115,059 & 118,160 & 117,049 & 121,380 & 122,381 & $103(0.09 \%)$ & $41(0.03 \%)$ & $13(0.01 \%)$ & $1(0.00 \%)$ & $0(0.00 \%)$ \\
\hline Helse Bergen HF & Large & 376,996 & 388,058 & 409,798 & 432,519 & 448,597 & $0(0.00 \%)$ & $1(0.00 \%)$ & $2(0.00 \%)$ & $2(0.00 \%)$ & $0(0.00 \%)$ \\
\hline Helse Førde HF & Medium & 110,630 & 109,995 & 112,956 & 114,554 & 119,052 & $11(0.01 \%)$ & $2(0.00 \%)$ & $0(0.00 \%)$ & $0(0.00 \%)$ & $2(0.00 \%)$ \\
\hline Betanien Hospital (Hordaland) ${ }^{a}$ & Small & 1675 & 2059 & 2097 & 2104 & 2192 & $0(0.00 \%)$ & $0(0.00 \%)$ & $0(0.00 \%)$ & $0(0.00 \%)$ & $0(0.00 \%)$ \\
\hline Haugesund San. Revmatismesykehus ${ }^{a}$ & Small & 21,914 & 21,066 & 18,082 & 24,333 & 23,915 & $2(0.01 \%)$ & $0(0.00 \%)$ & $0(0.00 \%)$ & $0(0.00 \%)$ & $0(0.00 \%)$ \\
\hline Haraldsplass Diakonale Sykehus ${ }^{a}$ & Small & 17,036 & 17,187 & 19,269 & 20,082 & 21,466 & $0(0.00 \%)$ & $1(0.01 \%)$ & $0(0.00 \%)$ & $0(0.00 \%)$ & $0(0.00 \%)$ \\
\hline Central Norway & & 695,162 & 724,617 & 763,467 & 784,757 & 804,753 & $448(0.06 \%)$ & $23(0.00 \%)$ & $1(0.00 \%)$ & $0(0.00 \%)$ & $32(0.00 \%)$ \\
\hline St. Olavs Hospital HF & Large & 327,390 & 350,338 & 368,701 & 382,669 & 393,556 & $448(0.14 \%)$ & $23(0.01 \%)$ & $1(0.00 \%)$ & $0(0.00 \%)$ & $10(0.00 \%)$ \\
\hline Helse Nord-Trøndelag HF & Medium & 100,797 & 99,562 & 109,382 & 109,110 & 112,597 & $0(0.00 \%)$ & $0(0.00 \%)$ & $0(0.00 \%)$ & $0(0.00 \%)$ & $22(0.02 \%)$ \\
\hline Helse Møre og Romsdal HF & Large & 266,975 & 274,717 & 285,384 & 292,978 & 298,600 & $0(0.00 \%)$ & $0(0.00 \%)$ & $0(0.00 \%)$ & $0(0.00 \%)$ & $0(0.00 \%)$ \\
\hline Northern Norway & & 470,078 & 484,151 & 502,839 & 515,029 & 514,316 & $1739(0.37 \%)$ & $876(0.18 \%)$ & $986(0.20 \%)$ & $955(0.19 \%)$ & $991(0.19 \%)$ \\
\hline Helse Finnmark HF & Medium & 55,048 & 54,132 & 55,108 & 59,607 & 59,092 & $14(0.03 \%)$ & $33(0.06 \%)$ & $39(0.07 \%)$ & $105(0.18 \%)$ & $76(0.13 \%)$ \\
\hline Universitetssykehuset i Nord-Norge HF & Large & 214,538 & 227,831 & 235,486 & 238,232 & 241,248 & $1325(0.62 \%)$ & $780(0.34 \%)$ & $848(0.36 \%)$ & $558(0.23 \%)$ & $778(0.32 \%)$ \\
\hline Nordlandssykehuset HF & Medium & 122,723 & 126,532 & 130,953 & 132,566 & 133,766 & $147(0.12 \%)$ & $63(0.05 \%)$ & $99(0.08 \%)$ & $292(0.22 \%)$ & $137(0.10 \%)$ \\
\hline Helgelandssykehuset HF & Medium & 77,769 & 75,656 & 81,292 & 84,624 & 80,210 & $253(0.33 \%)$ & $0(0.00 \%)$ & $0(0.00 \%)$ & $0(0.00 \%)$ & $0(0.00 \%)$ \\
\hline South-Eastern Norway & & $2,573,532$ & $2,625,076$ & $2,711,593$ & $2,783,087$ & $2,819,054$ & $318(0.01 \%)$ & $41(0.00 \%)$ & $19(0.00 \%)$ & $159(0.01 \%)$ & $170(0.01 \%)$ \\
\hline Sunnaas sykehus HF & Small & 2691 & 3922 & 3598 & 3285 & 4388 & $0(0.00 \%)$ & $4(0.10 \%)$ & $5(0.14 \%)$ & $132(4.02 \%)$ & $154(3.51 \%)$ \\
\hline Vestre Viken HF & Large & 287,427 & 277,960 & 296,535 & 306,315 & 326,293 & $0(0.00 \%)$ & $3(0.00 \%)$ & $1(0.00 \%)$ & $0(0.00 \%)$ & $1(0.00 \%)$ \\
\hline Akershus universitetssykehus HF & Large & 175,830 & 185,536 & 233,530 & 254,194 & 248,798 & $0(0.00 \%)$ & $0(0.00 \%)$ & $0(0.00 \%)$ & $0(0.00 \%)$ & $0(0.00 \%)$ \\
\hline Sykehuset Innlandet HF & Large & 317,634 & 320,325 & 327,537 & 335,019 & 341,459 & $97(0.03 \%)$ & $14(0.00 \%)$ & $1(0.00 \%)$ & $4(0.00 \%)$ & $1(0.00 \%)$ \\
\hline Sykehuset Østfold HF & Large & 200,674 & 195,314 & 196,563 & 205,507 & 212,247 & $137(0.07 \%)$ & $5(0.00 \%)$ & $2(0.00 \%)$ & $3(0.00 \%)$ & $0(0.00 \%)$ \\
\hline Sørlandet sykehus HF & Large & 267,781 & 271,263 & 279,041 & 292,567 & 298,291 & $74(0.03 \%)$ & $15(0.01 \%)$ & $8(0.00 \%)$ & $18(0.01 \%)$ & $13(0.00 \%)$ \\
\hline Sykehuset i Vestfold HF & Large & 196,826 & 195,674 & 205,989 & 215,857 & 213,254 & $0(0.00 \%)$ & $0(0.00 \%)$ & $2(0.00 \%)$ & $1(0.00 \%)$ & $0(0.00 \%)$ \\
\hline Sykehuset Telemark HF & Medium & 155,306 & 164,000 & 169,598 & 173,197 & 154,658 & $0(0.00 \%)$ & $0(0.00 \%)$ & $0(0.00 \%)$ & $0(0.00 \%)$ & $0(0.00 \%)$ \\
\hline Oslo universitetssykehus HF & Large & 825,891 & 859,476 & 828,164 & 815,140 & 832,613 & $0(0.00 \%)$ & $0(0.00 \%)$ & $0(0.00 \%)$ & $1(0.00 \%)$ & $0(0.00 \%)$ \\
\hline Betanien Hospital (Telemark) ${ }^{a}$ & Small & 14,868 & 16,983 & 18,760 & 19,815 & 19,642 & $0(0.00 \%)$ & $0(0.00 \%)$ & $0(0.00 \%)$ & $0(0.00 \%)$ & $1(0.01 \%)$ \\
\hline Lovisenberg $^{a}$ & Medium & 43,071 & 45,088 & 52,065 & 53,489 & 57,058 & 10 (0.02\%) & $0(0.00 \%)$ & $0(0.00 \%)$ & $0(0.00 \%)$ & $0(0.00 \%)$ \\
\hline
\end{tabular}


Table 2 Outpatient visits and telemedicine consultations in the period 2009-2013 in the publicly funded hospitals in Norway (Continued)

\begin{tabular}{|c|c|c|c|c|c|c|c|c|c|c|c|}
\hline Martina Hansens hospital ${ }^{a}$ & Small & 22,934 & 22,964 & 25,021 & 29,528 & 29,568 & $0(0.00 \%)$ & $0(0.00 \%)$ & $0(0.00 \%)$ & $0(0.00 \%)$ & $0(0.00 \%)$ \\
\hline Revmatismesykehuset Lillehammer ${ }^{a}$ & Small & 10,701 & 10,803 & 12,351 & 13,916 & 13,960 & $0(0.00 \%)$ & $0(0.00 \%)$ & $0(0.00 \%)$ & $0(0.00 \%)$ & $0(0.00 \%)$ \\
\hline Diakonhjemmet ${ }^{a}$ & Medium & 51,898 & 55,768 & 62,841 & 65,258 & 66,825 & $0(0.00 \%)$ & $0(0.00 \%)$ & $0(0.00 \%)$ & $0(0.00 \%)$ & $0(0.00 \%)$ \\
\hline Total & & $4,618,683$ & $4,764,684$ & $4,925,202$ & $5,077,642$ & $5,165,586$ & 2745 (0.06 \%) & $1186(0.02 \%)$ & 1827 (0.04 \%) & 2700 (0.05 \%) & $2879(0.06 \%)$ \\
\hline
\end{tabular}

aprivate specialist health facilities

Values in brackets (\%) represent the percentage of telemedicine consultations compared to the number of outpatient visits, by year and hospital 


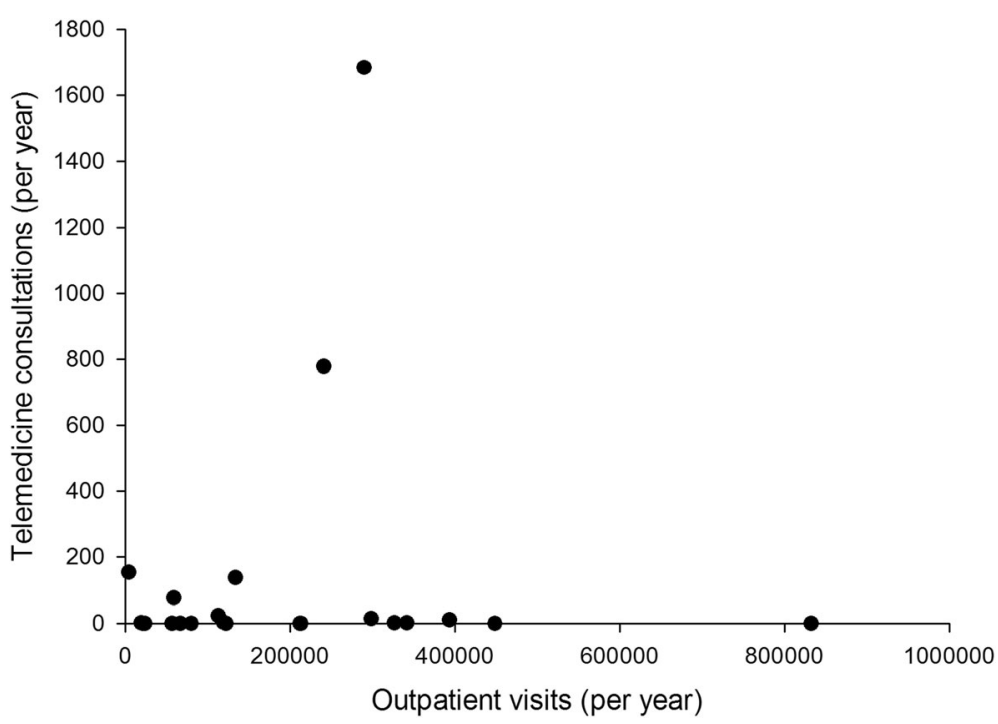

Fig. 3 Telemedicine usage compared to hospital size, expressed as outpatient visits in 2013

the proportion of telemedicine consultations over the total number of outpatient visits in 2013.

Neurosurgery and rehabilitation were the clinical specialties where telemedicine was used most, with a relative use in 2013 corresponding to $2.23 \%$ and $0.79 \%$, respectively. Neurosurgery can be considered as a clinical specialty with a low activity, which appears to be suitable to the use of telemedicine to deliver visits remotely. Early in 2009 over $5 \%$ of all outpatient visits in neurosurgery were delivered via videoconferencing. The use decreased during the following years. Rehabilitation is a clinical specialty with a high level of activity in terms of outpatient visits.
Looking at the number of telemedicine consultations in this field, there was a steady growth over the 5 years, and the level in 2013 was almost 4 times higher than in 2009. Rehabilitation became largely the most common clinical specialty in telemedicine. Apart from neurosurgery and rehabilitation, only six other clinical specialties recorded more than 100 telemedicine consultations. These included eye diseases, endocrinology, cardiovascular diseases, neurology, children's diseases, and skin and venereal diseases. All these specialties, however, experienced a decline in the number of telemedicine consultations occurred from 2009 to 2013.

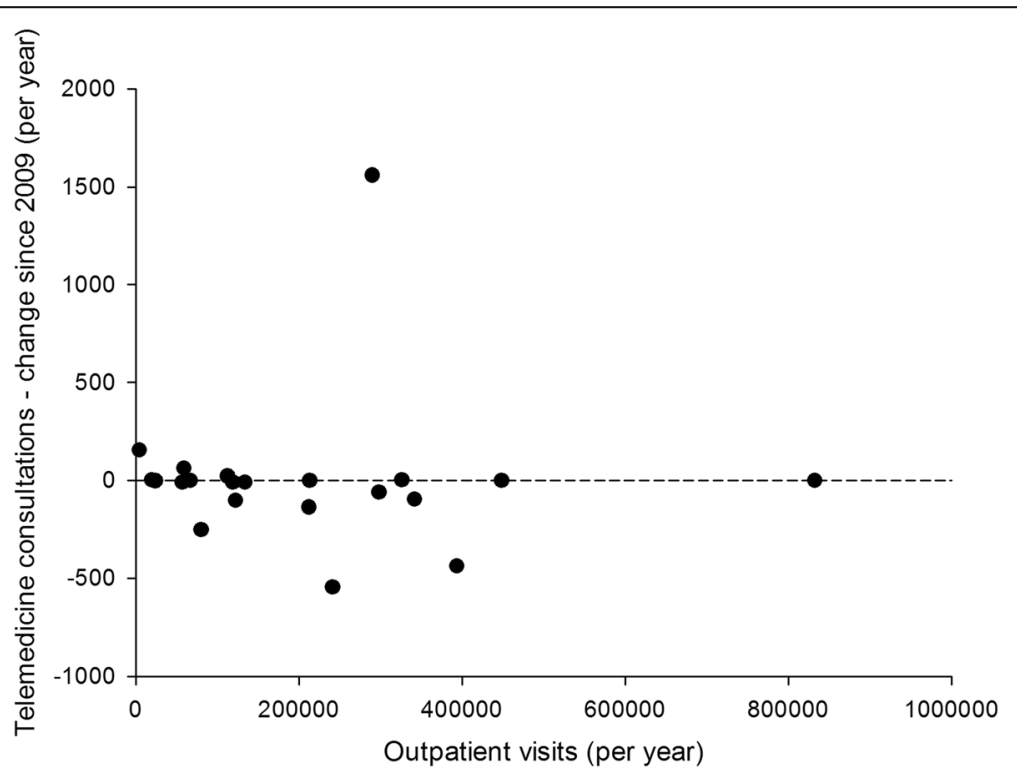

Fig. 4 Telemedicine growth compared to hospital size, expressed as outpatient visits in 2013 
Table 3 Outpatient visits and telemedicine consultations in the period 2009-2013 in the different clinical specialties

\begin{tabular}{|c|c|c|c|c|c|c|c|c|c|c|c|}
\hline Clinical specialty & Activity & $\begin{array}{l}\text { Outpatient } \\
\text { visits (2009) }\end{array}$ & $\begin{array}{l}\text { Outpatient } \\
\text { visits (2010) }\end{array}$ & $\begin{array}{l}\text { Outpatient } \\
\text { visits (2011) }\end{array}$ & $\begin{array}{l}\text { Outpatient } \\
\text { visits (2012) }\end{array}$ & $\begin{array}{l}\text { Outpatient } \\
\text { visits (2013) }\end{array}$ & $\begin{array}{l}\text { Telemedicine } \\
\text { consultations } \\
\text { (2009) }\end{array}$ & $\begin{array}{l}\text { Telemedicine } \\
\text { consultations } \\
(2010)\end{array}$ & $\begin{array}{l}\text { Telemedicine } \\
\text { consultations } \\
\text { (2011) }\end{array}$ & $\begin{array}{l}\text { Telemedicine } \\
\text { consultations } \\
\text { (2012) }\end{array}$ & $\begin{array}{l}\text { Telemedicine } \\
\text { consultations } \\
\text { (2013) }\end{array}$ \\
\hline Neurosurgery & Small & 14,701 & 16,858 & 19,144 & 20,401 & 21,037 & $803(5.46 \%)$ & $384(2.28 \%)$ & $469(2.45 \%)$ & $274(1.34 \%)$ & $469(2.23 \%)$ \\
\hline Rehabilitation & High & 162,434 & 174,216 & 194,161 & 203,567 & 198,229 & $389(0.24 \%)$ & $162(0.09 \%)$ & 789 (0.41 \%) & $1719(0.84 \%)$ & $1853(0.93 \%)$ \\
\hline Eye diseases & High & 228,680 & 266,363 & 287,130 & 301,316 & 311,324 & 154 (0.07 \%) & $137(0.05 \%)$ & $231(0.08 \%)$ & $229(0.08 \%)$ & $291(0.09 \%)$ \\
\hline Endocrinology & Medium & 108,866 & 117,577 & 119,423 & 125,502 & 130,146 & 16 (0.01\%) & $2(0.00 \%)$ & $5(0.00 \%)$ & $23(0.02 \%)$ & $26(0.02 \%)$ \\
\hline Cardiovascular diseases & High & 222,183 & 228,739 & 249,516 & 260,106 & 263,619 & $152(0.07 \%)$ & $58(0.03 \%)$ & $51(0.02 \%)$ & 30 (0.01\%) & $42(0.02 \%)$ \\
\hline Obstetrics & High & 482,661 & 502,400 & 513,143 & 508,829 & 510,189 & $102(0.02 \%)$ & $18(0.00 \%)$ & $14(0.00 \%)$ & 87 (0.02 \%) & 71 (0.01\%) \\
\hline Digestive diseases & Medium & 122,480 & 142,525 & 162,385 & 174,526 & 175,016 & $23(0.02 \%)$ & $1(0.00 \%)$ & $11(0.01 \%)$ & $31(0.02 \%)$ & $23(0.01 \%)$ \\
\hline Pulmonary diseases & Medium & 100,842 & 112,729 & 118,963 & 121,942 & 124,625 & 16 (0.02 \%) & $4(0.00 \%)$ & $20(0.02 \%)$ & $16(0.01 \%)$ & $16(0.01 \%)$ \\
\hline Plastic surgery & Medium & 52,588 & 55,257 & 59,980 & 56,998 & 63,413 & $0(0.00 \%)$ & $1(0.00 \%)$ & $3(0.01 \%)$ & $0(0.00 \%)$ & $7(0.01 \%)$ \\
\hline Kidney diseases & Medium & 53,312 & 52,288 & 54,164 & 57,058 & 58,516 & 12 (0.02 \%) & $3(0.01 \%)$ & $3(0.01 \%)$ & $11(0.02 \%)$ & $6(0.01 \%)$ \\
\hline Urology & Medium & 135,833 & 148,557 & 160,630 & 166,324 & 170,295 & 49 (0.04 \%) & $9(0.01 \%)$ & $17(0.01 \%)$ & $32(0.02 \%)$ & 15 (0.01\%) \\
\hline Neurology & Medium & 143,640 & 150,588 & 184,850 & 170,295 & 170,908 & $369(0.26 \%)$ & 12 (0.01\%) & 20 (0.01\%) & $32(0.02 \%)$ & 12 (0.01\%) \\
\hline General surgery & Medium & 166,569 & 146,575 & 133,809 & 128,941 & 120,954 & 35 (0.02 \%) & 10 (0.01\%) & $7(0.01 \%)$ & 16 (0.01\%) & $4(0.00 \%)$ \\
\hline Children's diseases & High & 212,285 & 215,727 & 198,207 & 219,036 & 218,822 & $120(0.06 \%)$ & 25 (0.01\%) & $5(0.00 \%)$ & 11 (0.01\%) & $7(0.00 \%)$ \\
\hline Orthopaedic surgery & High & 647,839 & 708,595 & 739,050 & 744,014 & 782,384 & $60(0.01 \%)$ & $72(0.01 \%)$ & 76 (0.01\%) & $139(0.02 \%)$ & $23(0.00 \%)$ \\
\hline Oncology and radiotherapy & High & 94,416 & 196,456 & 206,934 & 229,263 & 239,773 & $71(0.08 \%)$ & $9(0.00 \%)$ & $2(0.00 \%)$ & $6(0.00 \%)$ & $7(0.00 \%)$ \\
\hline Skin and venereal diseases & High & 197,707 & 204,552 & 189,415 & 222,658 & 216,227 & $220(0.11 \%)$ & $239(0.12 \%)$ & $93(0.05 \%)$ & $9(0.00 \%)$ & $5(0.00 \%)$ \\
\hline Gastroenterological surgery & Medium & 114,129 & 123,111 & 126,741 & 130,506 & 141,967 & $9(0.01 \%)$ & $4(0.00 \%)$ & $1(0.00 \%)$ & $25(0.02 \%)$ & $2(0.00 \%)$ \\
\hline Anaesthesiology & Small & 41,515 & 44,411 & 36,567 & 47,811 & 47,342 & $2(0.00 \%)$ & $3(0.01 \%)$ & $1(0.00 \%)$ & $0(0.00 \%)$ & $0(0.00 \%)$ \\
\hline Haematology & Medium & 61,811 & 75,488 & 84,666 & 91,659 & 94,146 & $14(0.02 \%)$ & $0(0.00 \%)$ & $0(0.00 \%)$ & $0(0.00 \%)$ & $0(0.00 \%)$ \\
\hline Cardiovascular surgery & Medium & 43,410 & 47,502 & 53,060 & 54,176 & 53,752 & $1(0.00 \%)$ & $1(0.00 \%)$ & $3(0.01 \%)$ & $4(0.01 \%)$ & $0(0.00 \%)$ \\
\hline Ear, nose and throat diseases & High & 324,964 & 333,776 & 353,326 & 356,885 & 365,251 & 15 (0.00\%) & $9(0.00 \%)$ & $3(0.00 \%)$ & $2(0.00 \%)$ & $0(0.00 \%)$ \\
\hline General internal medicine & Medium & 68,642 & 65,136 & 61,923 & 53,627 & 55,204 & 29 (0.04 \%) & $2(0.00 \%)$ & $1(0.00 \%)$ & $0(0.00 \%)$ & $0(0.00 \%)$ \\
\hline Geriatrics & Small & 16,338 & 16,931 & 18,427 & 19,370 & 18,797 & $10(0.06 \%)$ & $0(0.00 \%)$ & $0(0.00 \%)$ & $2(0.01 \%)$ & $0(0.00 \%)$ \\
\hline Infectious diseases & Small & 28,603 & 31,889 & 34,297 & 38,322 & 39,646 & $1(0.00 \%)$ & $0(0.00 \%)$ & $0(0.00 \%)$ & $1(0.00 \%)$ & $0(0.00 \%)$ \\
\hline Maxillofacial and mouth disease & Small & 29,634 & 27,554 & 26,746 & 28,005 & 29,006 & $10(0.03 \%)$ & $2(0.01 \%)$ & $0(0.00 \%)$ & $0(0.00 \%)$ & $0(0.00 \%)$ \\
\hline Other clinical specialities & High & 622,864 & 410,120 & 367,172 & 365,899 & 358,590 & $61(0.01 \%)$ & $18(0.00 \%)$ & $2(0.00 \%)$ & $0(0.00 \%)$ & $0(0.00 \%)$ \\
\hline Pregnancy/parathyroid surgery & Small & 2171 & 9883 & 15,717 & 17,186 & 21,182 & 0 (0.00 \%) & $0(0.00 \%)$ & 0 (0.00 \%) & 0 (0.00 \%) & $0(0.00 \%)$ \\
\hline Rheumatology & Medium & 117,566 & 138,881 & 155,656 & 163,420 & 165,226 & $2(0.00 \%)$ & $1(0.00 \%)$ & $0(0.00 \%)$ & $1(0.00 \%)$ & $0(0.00 \%)$ \\
\hline Total & & $4,618,683$ & $4,764,684$ & $4,925,202$ & $5,077,642$ & $5,165,586$ & $2745(0.06 \%)$ & $1186(0.02 \%)$ & $1827(0.04 \%)$ & $2700(0.05 \%)$ & $2879(0.06 \%)$ \\
\hline
\end{tabular}

Values in brackets (\%) represent the percentage of telemedicine consultations compared to the number of outpatient visits, by year and clinical specialty 


\section{International comparison}

Table 4 summarises data from eight different telemedicine networks providing consultations in multiple specialties [17, 22-28] in addition to the data from Norway. The pro capita rate of telemedicine varied from about 1 consultation per year per 1000 persons to over 20 in the largest and well-established telemedicine networks. Figure 5 compares the level of activity in the different statewide networks to the size of the population served by each network. It is apparent that the larger is the population served, the larger is the telemedicine network in terms of sites, and the higher is the telemedicine service usage. This might be explained by the presence of economies of scale. The data can be fitted by a sigmoid curve. While most of the telemedicine networks still have a lower level of activity, the Veterans Health Administration Telehealth Network [22], the Ontario Telemedicine Network [17] and the Alaska Federal Health Care Access Network [26] seem to have succeeded in scaling up both adoption and use of telemedicine. The large telemedicine operations employ both store-and-forward technology and videoconferencing.

\section{Discussion}

\section{Overall trend of telemedicine}

The present paper reports unique statewide data on the routine use of telemedicine in Norwegian hospitals over a 5 -year period. The number of telemedicine consultations increased and followed a similar trend to that of outpatient visits. This presumably reflects the increase of the population and their health needs. An overall growing trend in the delivery of telemedicine has been described in other studies reporting statewide or network-based data over time. Since 1994, telemedicine has become an integral part of the Veterans Health Administration in the United States. Data show a continuous increase in the number of veterans served by telemedicine over 20 years as well as in the number of telemedicine consultations. Telemedicine activity followed an S-shaped innovation curve [22], confirming the hypothesis that telemedicine adoption follows the growth curve typical of health technologies and other innovations [6]. The Ontario Telemedicine Network, the largest telemedicine service provider in Canada and one of the largest in the world, facilitates access to medical care in areas that are often underserved. The number of telemedicine consultations increased in all four Ontario regions from 2008 to 2013, with higher rates in rural North Ontario [17]. The Municipal Department of Health of Belo Horizonte, Brazil, established a telemedicine program in which specialist support was offered to primary care providers. The number of store-and-forward consultations grew from 2006 to 2009 [29]. The African Francophone Telemedicine Network was established to improve access to medical care in the rural Altiplano region of Bolivia, serving a population of about 200,000 inhabitants. The number of telemedicine consultations increased from 2011 to 2013, reaching a yearly average of 700 consultations [23].

\section{Activity decline in $\mathbf{2 0 1 0}$}

The data from Norwegian hospitals showed a considerable reduction of telemedicine consultations in 2010 . This observed decline might be due to organisational factors [29], such as lack of resources [30], or state-level policies, including reimbursement [31, 32]. In 2009 the Norwegian Health Network was established to provide an infrastructure for secure communication in the health

Table 4 Comparison of telemedicine activity among nine different statewide networks delivering multispecialty services

\begin{tabular}{|c|c|c|c|c|c|c|c|}
\hline & Reference & Technology & Year & Network size & $\begin{array}{l}\text { Population } \\
\text { served }\end{array}$ & $\begin{array}{l}\text { Telemedicine } \\
\text { consultations }\end{array}$ & Pro capita rate \\
\hline $\begin{array}{l}\text { Veterans Health } \\
\text { Administration, USA }\end{array}$ & [Darkins 2014] [22] & VC and SF & 2013 & $\begin{array}{l}152 \text { Medical Centers, } 600 \\
\text { community-based outpatient } \\
\text { clinics, patients' homes }\end{array}$ & $21,600,000$ & 600,000 & 27.8 \\
\hline Alaska, USA & [Kokesh 2011] [26] & VC and SF & 2009 & $\begin{array}{l}248 \text { sites, more than } 700 \\
\text { health-care providers }\end{array}$ & 700,000 & 14,000 & 20.0 \\
\hline Ontario, Canada & [O'Gorman 2015] [17] & VC and SF & 2013 & 2026 sites & $13,550,900$ & 221,353 & 16.3 \\
\hline $\begin{array}{l}\text { African Francophone } \\
\text { Telemedicine Network, } \\
\text { Bolivia }\end{array}$ & [Vargas 2014] [23] & VC and SF & 2013 & more than 20 health institutions & 200,000 & 700 & 3.5 \\
\hline Alberta, Canada & [Ohinmaa 2006] [24] & VC & 2003 & 212 sites & $3,000,000$ & 5766 & 1.9 \\
\hline Georgia, USA & [Brewer 2011] [25] & VC and SF & 2009 & 51 statewide access points & $9,829,211$ & 18,000 & 1.8 \\
\hline Nebraska, USA & [Meyers 2012] [27] & Mainly VC & 2010 & over 110 sites & $1,800,000$ & 2600 & 1.4 \\
\hline Western Australia & [Dillon 2005] [28] & VC & 2003 & 104 sites & $2,000,000$ & 2151 & 1.1 \\
\hline Norway & [present study] & VC & 2013 & 28 hospitals & $5,165,802$ & 2879 & 0.6 \\
\hline
\end{tabular}

apro capita rate: consultations/1000 inhabitants 


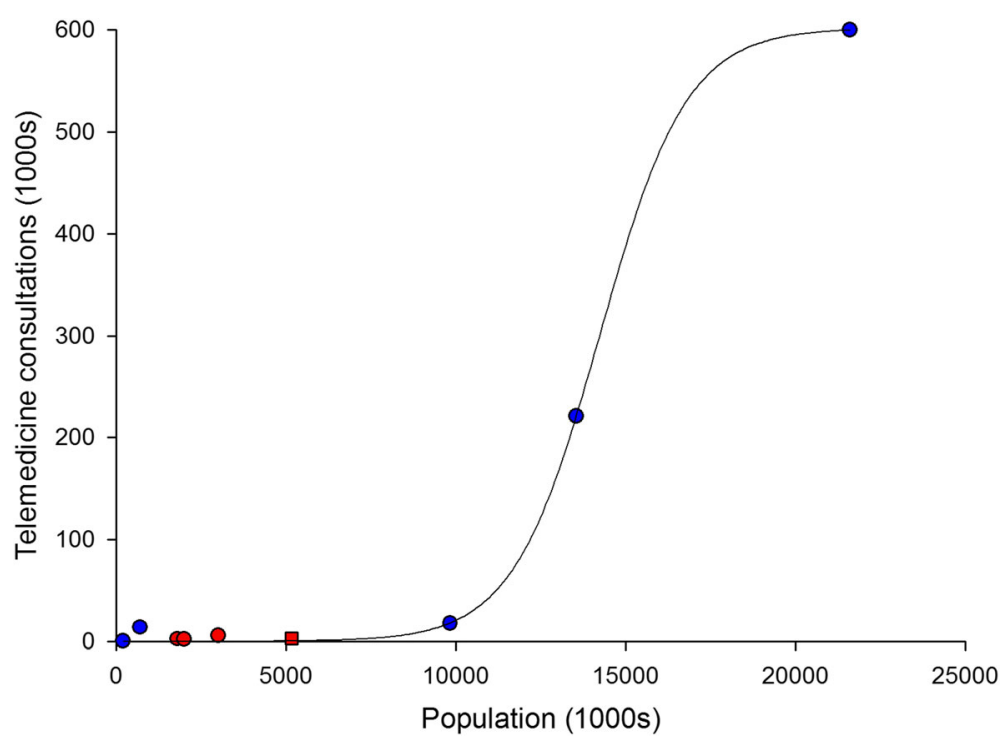

Fig. 5 Telemedicine activity in nine statewide networks compared to the population served. The fitted line is a sigmoid. Networks mainly using videoconferencing are shown with red symbols; networks using both videoconferencing and store-and-forward telemedicine are shown with blue symbols. The square symbol represents Norway

sector in Norway. The implementation and temporary transition to this statewide network might explain the decline of telemedicine in 2010. Another factor to be considered is the nature of the telemedicine consultations. Similar studies showed increased use of storeand-forward consultations over time, while real-time consultations via videoconferencing become less frequent [33, 34]. In 1996, Norway became the first country to implement an official telemedicine fee, without distinction between video and still image solutions [14]. In 2008, however, reimbursement for store-and-forward telemedicine was discontinued, and only telemedicine consultations performed via videoconferencing were reimbursed. While store-and-forward telemedicine appears to be efficient and suitable in routine clinical practice, a lack of reimbursement represents a barrier to its use. We believe that a revision of the current reimbursement policies might create incentives which would result in a wider use of telemedicine by Norwegian hospitals.

\section{Adoption of telemedicine}

Adoption at the regional level was $100 \%$, that is, use of telemedicine consultations was reported in all four health regions during the period 2009-2013. The results confirm the hypothesis that telemedicine is mainly used to increase access to healthcare services in remote areas with underserved population. Twenty-one out of 28 hospitals reported using telemedicine, i.e. there was $75 \%$ adoption at the hospital level. Thus adoption of telemedicine by Norwegian hospitals appeared to be high, both at regional level and at institutional level. Only a minority of late potential users [19] have still to adopt telemedicine. All the four hospitals in Northern Norway were active in delivering telemedicine over the 5-year study. This might be explained by the higher needs for delivering services remotely due to barriers related to distance and transportation difficulties such as in Northern Norway. Most of the hospitals delivering telemedicine were based in regions characterised by higher remoteness. Adoption rate by percentage of physicians who used the store-and-forward consultations in Belo Horizonte, Brazil, reached $6 \%$ at network level and $18.5 \%$ at district level. Of the adopting physicians, some stopped using telemedicine, while a few remained responsible for most telemedicine consultations [29]. All 21 geographical regions from the Veterans Health Administration Telehealth Network used teledermatology in 2014, with 4 of them collectively reporting $51 \%$ of the patient encounters [34]. The presence of "heavy users" is confirmed by the data from Norway, where only a few hospitals delivered more than 50 telemedicine consultations per year. In the United States, the distribution of telemedicine-related costs covered by Medicare varies across states, services, and specialties. This suggests that factors other than simply rurality or need have driven adoption [35].

\section{Use of telemedicine}

Despite the growing trend and the high adoption, the relative use of telemedicine compared to that of outpatient visits was low. Hospitals in Norway therefore appear not ready yet to replace a substantial proportion of outpatient face-to-face visits with remote consultations. Medicare has been a key payer for telemedicine in 
the United States since late 1990s, but telemedicinerelated costs remain a relatively miniscule part of overall expenditures [35]. A recent report identified six important prerequisites for successful implementation of telemedicine: 1) the national plans exist, but are not well enough coordinated and not supported by sufficient resources; 2 ) access to a secure communications infrastructure is to a great extent in place; 3) the use of standards is not mandatory; 4) the implementation of Electronic Health Records is very good, but interoperability should be improved; 5) laws should be adapted to the modern way of working; and 6) reimbursement for new ways of health service delivery is not in place [36]. Norway still has some way to go in its use of telemedicine. For example, if it had the same pro capita rate of telemedicine usage as in the Ontario Telemedicine Network [17], the use of routine telemedicine in Norway would increase from 2879 to 78,213 telemedicine consultations every year, almost 30 times more than the current value.

Telemedicine can be used to replace referrals to an outpatient clinic [37], thus reducing travel [8] and unnecessary hospital accesses [38], especially to those living in remote areas. However, it is difficult to estimate the proportion of outpatient visits which could be potentially replaced with telemedicine consultations, since there have been no reports to date of the largescale use of outpatient telemedicine. It is unlikely that all outpatient visits in all specialties can be replaced by telemedicine visits. On the other hand, there is evidence that in some specialties, substantial numbers of visits can be avoided. Wootton et al. estimated that approximately half of all outpatient visits could be avoided in dermatology [8]. Jaatinen et al. found that a similar proportion of internal medicine and geriatric visits could be avoided in Finland [37]. McGill et al. found that $13 \%$ of visits to a rural fracture clinic in Queensland could be saved by use of telemedicine [39]. If telemedicine was used in just $10 \%$ of all outpatient visits in Norway, this would equate to about 500,000 telemedicine consultations per year, suggesting that there is room for about 100 times as many telemedicine consultations in the future.

\section{Conclusions}

We examined telemedicine adoption in Norway, exploring its level of utilisation overall, by health region, hospital, and clinical specialty. Adoption of telemedicine is Norway is high, with all the health regions and most of the hospitals reporting using telemedicine. The hospitals delivering telemedicine are mostly based in regions characterised by lower centrality and population density. Use of telemedicine has increased over the past five years. However, its relative use compared to the number of outpatient visits is still low. An international comparison shows that only few statewide telemedicine networks seem to have succeeded in scaling up both adoption and use of telemedicine. The present study provides new insights regarding the uptake of routine telemedicine delivered in a large scale. To facilitate future comparisons we recommend reporting data on adoption and utilisation over time from other statewide or network-based telemedicine services.

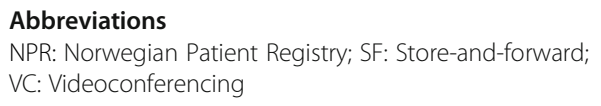

\section{Funding}

We acknowledge the Northern Norway Regional Health Authority for financial support in collecting data from the Norwegian Patient Registry.

Availability of data and materials

The datasets analysed during the current study are available from the corresponding author on reasonable request.

\section{Authors' contributions}

PZ and RW jointly initiated the study, analysed the data and interpreted the results. Both authors wrote, read and approved the final manuscript.

\section{Competing interests}

The authors declare that they have no competing interests.

\section{Consent for publication}

Not applicable.

\section{Ethics approval and consent to participate}

Not applicable.

\section{Author details}

${ }^{1}$ Norwegian Centre for E-health Research, University Hospital of North Norway, P.O. Box 359038 Tromsø, Norway. ${ }^{2}$ Faculty of Health Sciences, The Arctic University of Norway, Langnes, P.O. Box 60509037 Tromsø, Norway.

Received: 6 December 2015 Accepted: 13 September 2016

Published online: 20 September 2016

\section{References}

1. Doarn CR, Merrell RC. A roadmap for telemedicine: barriers yet to overcome Telemed J E Health. 2008;14(9):861-2.

2. Taylor J, Coates E, Wessels B, Mountain G, Hawley MS. Implementing solutions to improve and expand telehealth adoption: participatory action research in four community healthcare settings. BMC Health Serv Res. 2015;15(1):529.

3. Ekeland AG, Bowes A, Flottorp S. Effectiveness of telemedicine: a systematic review of reviews. Int J Med Inform. 2010;79(11):736-71.

4. Wootton R. Twenty years of telemedicine in chronic disease managementan evidence synthesis. J Telemed Telecare. 2012;18(4):211-20.

5. Mistry H. Systematic review of studies of the cost-effectiveness of telemedicine and telecare. Changes in the economic evidence over twenty years. J Telemed Telecare. 2012;18(1):1-6.

6. Zanaboni P, Wootton R. Adoption of telemedicine: from pilot stage to routine delivery. BMC Med Inform Decis Mak. 2012;12:1.

7. García-Lizana F, Giorgo F. The future of e-health, including telemedicine and telecare, in the European Union: from stakeholders' views to evidence based decisions. J Telemed Telecare. 2012;18(6):365-6.

8. Wootton R, Bahaadinbeigy K, Hailey D. Estimating travel reduction associated with the use of telemedicine by patients and healthcare professionals: proposal for quantitative synthesis in a systematic review. BMC Health Serv Res. 2011;11:185. 
9. Whitten P, Holtz B, Nguyen L. Keys to a successful and sustainable telemedicine program. Int J Technol Assess Health Care. 2010;26:211-6.

10. Ringard $\AA$, Sagan A, Sperre Saunes I, Lindahl AK. Norway: Health system review. Health Syst Transit. 2013;15(8):1-162.

11. Norwegian Directorate of Health. Norway and Health an Introduction. Oslo: Norwegian Directorate of Health; 2012.

12. Hansen $\mathrm{AH}$, Halvorsen PA, Ringberg U, Førde $\mathrm{OH}$. Socio-economic inequalities in health care utilisation in Norway: a population based crosssectional survey. BMC Health Serv Res. 2012;12:336.

13. Elford DR. Telemedicine in northern Norway. J Telemed Telecare. 1997;3(1):1-22.

14. Uldal SB. A survey of Norwegian telemedicine. J Telemed Telecare. 1999;5:32-7.

15. Norum J, Pedersen S, Størmer J, Rumpsfeld M, Stormo A, Jamissen N, Sunde $\mathrm{H}$, Ingebrigtsen $\mathrm{T}$, Larsen ML. Prioritisation of telemedicine services for large scale implementation in Norway. J Telemed Telecare. 2007;13(4):185-92.

16. Zanaboni $\mathrm{P}$, Knarvik U, Wootton R. Adoption of routine telemedicine in Norway: the current picture. Glob Health Action. 2014;7:22801.

17. O'Gorman LD, Hogenbirk JC, Warry W. Clinical telemedicine utilization in Ontario over the Ontario telemedicine network. Telemed J E Health. 2015. [Epub ahead of print].

18. Sosial-og helsedirektoratet. Innsatsstyrt finansiering 2015. Oslo: Helsedirektoratet; 2014

19. Rogers ME. Diffusion of innovation. New York: Free Press; 2003.

20. Kommunal- og moderniseringsdepartementet, Distriktsindeksen 2015 metode, datakilder og kvalitetssikring. Oslo; 2015.

21. Mars M, Scott R. Telemedicine service use: a new metric. J Med Internet Res. 2012;14(6):e178

22. Darkins A. The growth of telehealth services in the Veterans Health Administration between 1994 and 2014: a study in the diffusion of innovation. Telemed J E Health. 2014;20(9):761-8.

23. Vargas A, Ugalde M, Vargas R, Narvaez R, Geissbuhler A. Telemedicine in Bolivia: RAFT-Altiplano project, experiences, future prospects, and recommendations. Rev Panam Salud Publica. 2014:35(5-6):359-64.

24. Ohinma A, Scott R. A costing model for videoconferencing in Alberta. J Telemed Telecare. 2006;12(7):363-9.

25. Brewer R, Goble G, Guy P. A peach of a telehealth program: Georgia connects rural communities to better healthcare. Perspect Health Inf Manag. 2011:8:1c

26. Kokesh J, Ferguson AS, Patricoski C. The Alaska experience using store-andforward telemedicine for ENT care in Alaska. Otolaryngol Clin North Am. 2011:44:1359-74.

27. Meyers L, Gibbs D, Thacker M, Lafile L. Building a telehealth network through collaboration: the story of the Nebraska Statewide Telehealth Network. Crit Care Nurs Q. 2012;35:346-52.

28. Dillon E, Loermans J, Davis D, Xu C. Evaluation of the Western Australian Department of Health telehealth project. J Telemed Telecare. 2005;11 Suppl 2:S19-21.

29. Ruas SS, Assunção AÁ. Teleconsultations by primary care physicians of Belo Horizonte: challenges in the diffusion of innovation. Telemed J E Health. 2013;19(5):409-14.

30. Harvey S, Peterkin G, Wootton R. Eleven years of experience with lowbandwidth telemedicine in a nurse-led rural clinic in Scotland. J Telemed Telecare. 2010;16(8):417-21.

31. Krupinski EA, Weinstein RS. Telemedicine in an academic center-the Arizona Telemedicine Program. Telemed J E Health. 2013:19(5):349-56.

32. Neufeld JD, Doarn CR, Aly R. State policies influence medicare telemedicine utilization. Telemed J E Health. 2015. [Epub ahead of print].

33. Krishnan A, Fuska M, Dixon R, Sable CA. The evolution of pediatric teleechocardiography: 15-year experience of over 10,000 transmissions. Telemed J E Health. 2014;20(8):681-6.

34. Landow SM, Oh DH, Weinstock MA. Teledermatology within the Veterans Health Administration, 2002-2014. Telemed J E Health. 2015;21(10):769-73.

35. Neufeld JD, Doarn CR. Telemedicine spending by medicare: a snapshot from 2012. Telemed J E Health. 2015;21(8):686-93.

36. Hartvigsen G, Pedersen S. Lessons learned from 25 years with telemedicine in Northern Norway, Norwegian Centre for Integrated Care and Telemedicine. Tromsø: University Hospital of North Norway; 2015.

37. Jaatinen PT, Aarnio P, Remes J, Hannukainen J, Köymäri-Seilonen T. Teleconsultation as a replacement for referral to an outpatient clinic. J Telemed Telecare. 2002;8(2):102-6.
38. Zanaboni P, Scalvini S, Bernocchi P, Borghi G, Tridico C, Masella C. Teleconsultation service to improve healthcare in rural areas: acceptance, organizational impact and appropriateness. BMC Health Serv Res. 2009:9:238.

39. McGill A, North J. An analysis of an ongoing trial of rural videoconference fracture clinics. J Telemed Telecare. 2012:18(8):470-2.

\section{Submit your next manuscript to BioMed Central and we will help you at every step:}

- We accept pre-submission inquiries

- Our selector tool helps you to find the most relevant journal

- We provide round the clock customer support

- Convenient online submission

- Thorough peer review

- Inclusion in PubMed and all major indexing services

- Maximum visibility for your research

Submit your manuscript at www.biomedcentral.com/submit

) Biomed Central 